n.tronos

glyndwîn

Glyndŵr University

Glyndŵr University Research Online

Computing

Computer Science

$1-1-1999$

\title{
An independently fed log-periodic antenna for directed pulsed radiation
}

Peter S. Excell

Glyndwr University, p.excell@glyndwr.ac.uk

AD. Tinniswood

R W. Clarke

Follow this and additional works at: http://epubs.glyndwr.ac.uk/cair

Part of the Computer Engineering Commons, and the Computer Sciences Commons

\section{Recommended Citation}

Excell, P. S., Tinniswood, A. D., \& Clarke, R. W. (1999)'An independently fed log-periodic antenna for directed pulsed radiation'. IEEE Transactions on Electromagnetic Compatibility, 41(4), 344-349.

This Article is brought to you for free and open access by the Computer Science at Glyndŵr University Research Online. It has been accepted for inclusion in Computing by an authorized administrator of Glyndŵr University Research Online. For more information, please contact

d.jepson@glyndwr.ac.uk. 


\title{
An independently fed log-periodic antenna for directed pulsed radiation
}

\begin{abstract}
The development of an antenna capable of radiating a band-limited pulse with minimal distortion, negligible loss, and significant directivity is reported. A design was derived by modification of the conventional logperiodic dipole array to permit independent feeding of each dipole. This was modeled with a time-domain integral equation program and iterated to find a design that minimized phase dispersion across the operating band. The optimal design was realized in hardware, using a printed structure to feed the dipoles independently; this was located in the normal plane to prevent distortion of the radiated fields. When tested, the antenna was found to give a good quality radiated pulse in the main beam direction, with weaker and dispersed waveforms in other directions, indicating significant directive gain.
\end{abstract}

\section{Keywords}

Broad-band antenna, electromagnetic susceptibility, log-periodic dipole array, pulsed radiation, timedomain

\section{Disciplines}

Computer Engineering | Computer Sciences

\section{Comments}

C1999 IEEE. Personal use of this material is permitted. However, permission to reprint/republish this material for advertising or promotional purposes or for creating new collective works for resale or redistribution to servers or lists, or to reuse any copyrighted component of this work in other works must be obtained from the IEEE. This material is presented to ensure timely dissemination of scholarly and technical work. Copyright and all rights therein are retained by authors or by other copyright holders. All persons copying this information are expected to adhere to the terms and constraints invoked by each author's copyright. In most cases, these works may not be reposted without the explicit permission of the copyright holder. This paper was published in IEEE Transactions on Electromagnetic Compatibility in 1999. The definitive version is available at http:/ /ieeexplore.iee.org 


\title{
An Independently Fed Log-Periodic Antenna for Directed Pulsed Radiation
}

\author{
Peter S. Excell, Senior Member, IEEE, Adam D. Tinniswood, and Roger W. Clarke, Member, IEEE
}

\begin{abstract}
The development of an antenna capable of radiating a band-limited pulse with minimal distortion, negligible loss, and significant directivity is reported. A design was derived by modification of the conventional log-periodic dipole array to permit independent feeding of each dipole. This was modeled with a time-domain integral equation program and iterated to find a design that minimized phase dispersion across the operating band. The optimal design was realized in hardware, using a printed structure to feed the dipoles independently; this was located in the normal plane to prevent distortion of the radiated fields. When tested, the antenna was found to give a good quality radiated pulse in the main beam direction, with weaker and dispersed waveforms in other directions, indicating significant directive gain.
\end{abstract}

Index Terms - Broad-band antenna, electromagnetic susceptibility testing, log-periodic dipole array, pulsed radiation, timedomain integral equation method.

\section{INTRODUCTION}

$\mathbf{T}$ HE use of standard log-periodic dipoles for electromagnetic compatibility (EMC) testing is well established and it has also been demonstrated [1] that arrays of them can be used to generate near-field plane-wave test zones over larger volumes than are possible with a single antenna. In view of the predominance of impulsive interference in many practical situations and the trend toward wide-band radio communications systems, an investigation into the extension of the concept for generation of plane waves with a pulsed time-domain waveform was initiated.

The log-periodic antenna is attractive for use in wide-band arrays since being an end-fire structure, its physical aperture (normal to the main-beam direction) is smaller than is the case for many other designs. However, it is intuitively obvious that the standard form of the antenna will cause severe dispersion of the frequency components in a pulsed waveform. This is because the antenna is excited at the "nose" (the front end nearest to the shortest dipole element) and low-frequency components of the signal have to travel down the antenna structure until they reach a dipole that is near resonance. They then have to travel back the same distance as radiated fields before they can combine with the high-frequency components

Manuscript received September 28, 1998; revised July 29, 1999. This work was supported by the U.K. Engineering and Physical Sciences Research Council.

P. S. Excell and R. W. Clarke are with the Telecommunications Research Centre, University of Bradford, Bradford, West Yorkshire, BD7 1DP U.K.

A. D. Tinniswood was with Telecommunications Research Centre, University of Bradford, Bradford, West Yorkshire, BD7 1DP U.K. He is now with IBM Corporation, System Availability, Server Development 7TLNJA, Poughkeepsie, NY 12601 USA.

Publisher Item Identifier S 0018-9375(99)09403-X. radiated from short dipole elements near the nose. The result is that a time delay is imposed that is approximately proportional to the wavelength of the component.

Although it is somewhat counterintuitive, an earlier paper [2] had shown the feasibility of using log-periodic antennas to radiate pulses. However, this approach required the use of either a relatively long antenna with nonstandard element spacings or a chirped input signal to compensate for timedelays. The long antennas could pose difficulties in some circumstances and the generation of chirped input signals is difficult if a high-power test facility is desired, or if the antenna is required for wide-band communications. A search for smaller alternative designs capable of radiating a pulse without dispersion was thus undertaken.

\section{Simulation Studies Using Time-Domain INTEGRAL-EQUATION SOFTWARE}

\section{A. Trapezoidal Log-Periodic Antenna}

A time-domain integral-equation (TDIE) program was used to model log-periodic antennas under pulsed excitation without the need for Fourier transformation, as would be required with traditional frequency-domain programs [3]. The first antenna investigated was a trapezoidal-type log-periodic dipole array (LPDA) with a taper angle (the included angle of the loci of the tips of the elements) of $23^{\circ}$, which, for a given bandwidth, gives a shorter antenna than those reported in [2]. The scale factor was 0.88 and the spacing factor 0.15 - these values being chosen to give the antenna optimum conventional performance [4]. The resonant frequencies of the shortest and longest elements were $1 \mathrm{GHz}$ and $500 \mathrm{MHz}$, respectively, and the angle between the two halves of the antenna was $30^{\circ}$ (Fig. 1). When the antenna was excited with a 2 ns Gaussian pulse (Fig. 2), it was found that the radiated signal was much broader and contained several oscillations (Fig. 3), which are clearly the result of dispersion caused by the wavelengthdependent time delay in such a nose-fed antenna.

It was reasoned that if the exciting pulse at the input were replaced by a replica of the radiated waveform, but reversed in time, this would give a form of predistortion that would exactly compensate for the dispersive delays in the antenna. When this was tried in the software model a band-limited pulse of good quality resulted (Fig. 4). It should be noted that this radiating system is necessarily band-limited and, hence, radiation of pulses with a dc component is not possible: the radiated pulses should always have, at minimum, a positive and a negative swing to cancel the dc and the result in Fig. 4 


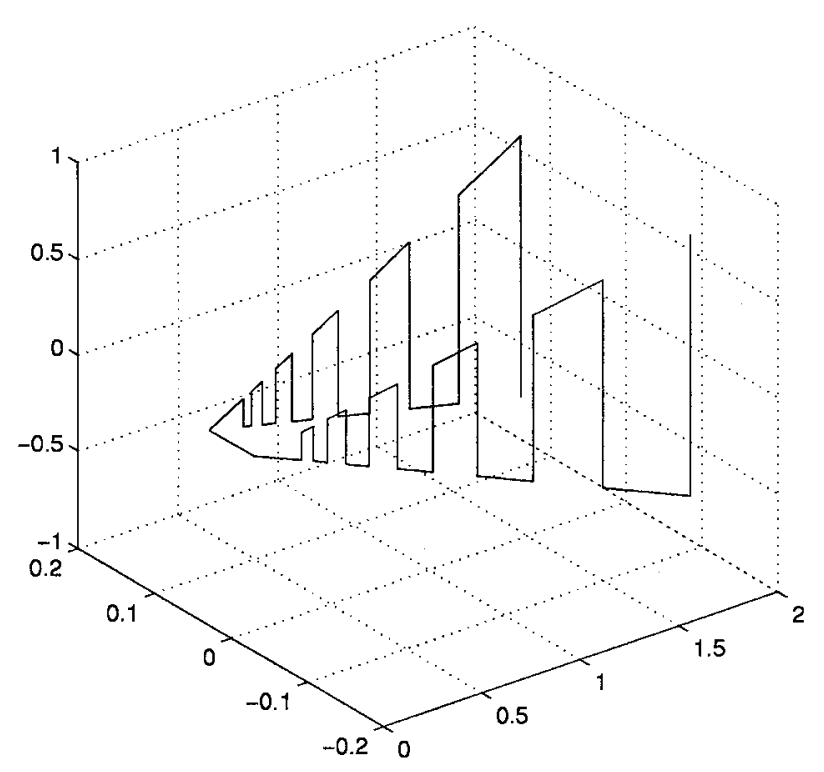

Fig. 1. The trapezoidal log-periodic antenna investigated with TDIE (the scales are in meters).

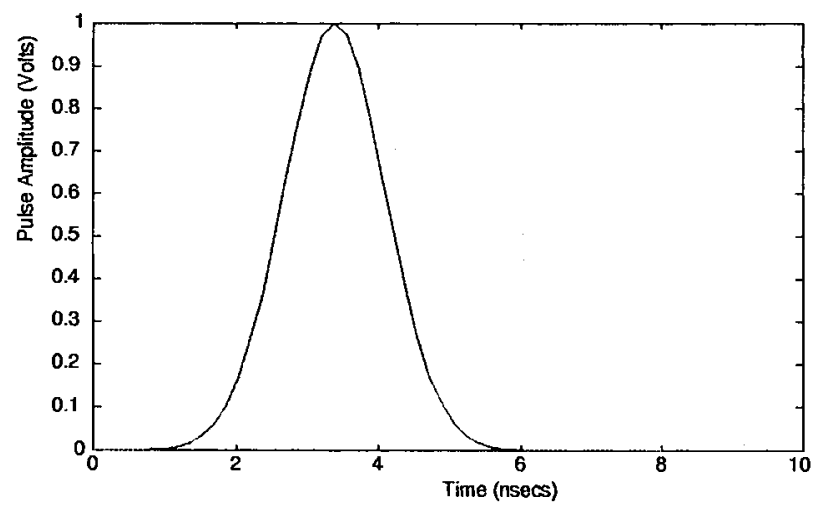

Fig. 2. The input pulse used in TDIE studies.

is a reasonable approximation to this. This approach could thus be used directly as a solution of the problem, but the cost of implementing the predistorted pulse could be substantial and the network to achieve it would be relatively inflexible (this solution is, in fact, closely similar to that of the chirped feed approach of [2]).

\section{B. Independently-Fed LPDA}

An investigation of further designs was thus instituted in an attempt to find a design that would accept a pulsed input and radiate it without dispersion, thus enabling the use of a simple pulse generator as the source. It was concluded that the traditional approach to feeding log-periodic antennas via a transmission line fed at the nose leads to unacceptable time delays in the elements more distant from the nose and that independent feeding of the elements should be tried. The standard practice of reversing the feed to alternate elements was retained as this gives the localized Yagi-like directive behavior of a LPDA.

A heuristic design procedure was adopted in which a standard log-periodic structure of eight solid rods was chosen

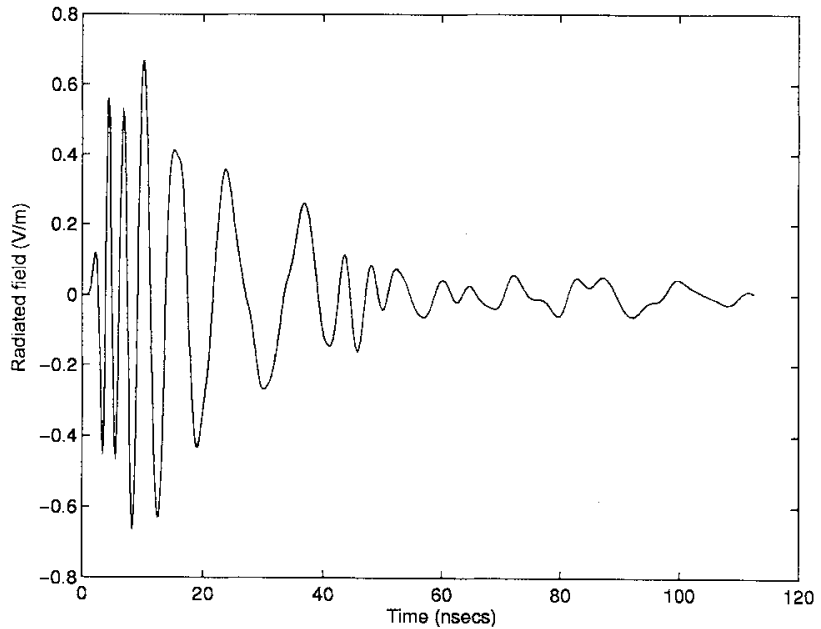

Fig. 3. TDIE predicted dominant component of electric field (i.e., vertically polarized in the sense of Fig. 1) radiated from the trapezoidal antenna at a distance of $10 \mathrm{~m}$ from the feed point in the main-beam direction when excited by the pulse in Fig. 2.

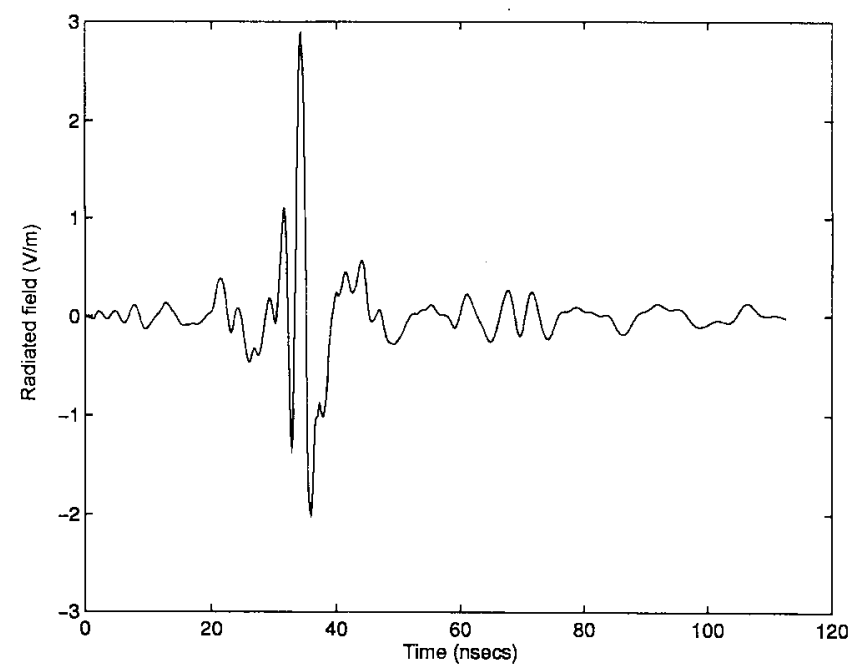

Fig. 4. TDIE predicted radiated pulse from trapezoidal antenna with predistorted input waveform. (Dominant component of electric field at a distance of $10 \mathrm{~m}$ from the feed point in the main-beam direction).

for study (Fig. 5). The lengths of the longest and shortest rods were 300 and $123 \mathrm{~mm}$, respectively, and their diameters were chosen to keep the length to diameter ratio approximately constant, as is required in an ideal LPDA. The excitation was, again, a 2 ns Gaussian pulse. In the first step, the field radiated was found when the shortest rod (number 1) was excited as a dipole with a voltage source at its center (Fig. 6). The same excitation was then applied simultaneously to a rod at the other end of the distribution (number 7), and the feed-point time delays for the two dipoles were adjusted to give maximized amplitude in the pair of superposed radiated pulses (Fig. 7). This procedure was continued for the full set of eight elements with several values of the included taper angle. After several iterations, a design was derived which showed relatively nondispersive radiation of a band-limited pulse from a 2 ns Gaussian input (Fig. 8). The optimum design was found to have a very small value of the spacing factor, giving an unusually large taper angle of $90^{\circ}$. The relative time delays in 


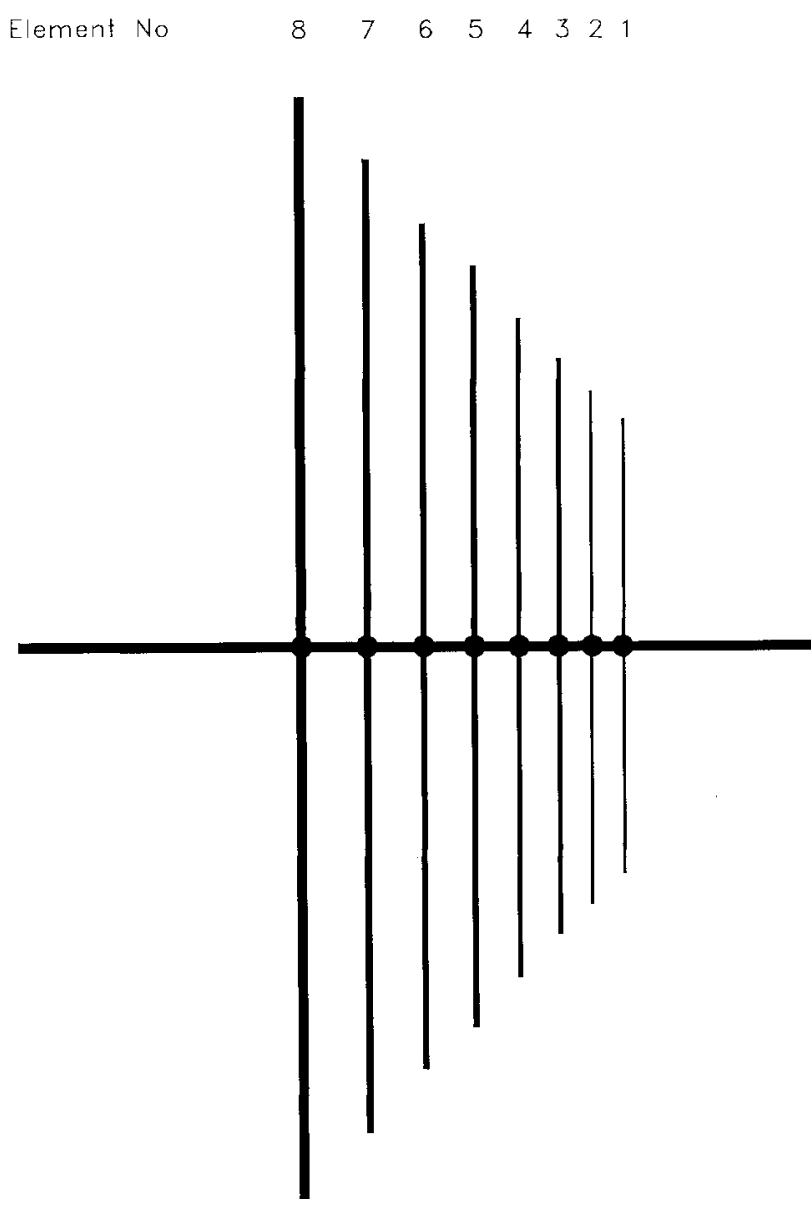

Fig. 5. The set of eight rods forming the independently-fed log-periodic antenna, as modeled by TDIE (for dimensions see Table I: the central support structure was not included in the model as it is normal to the electric field). The large taper angle used in the final design is shown here, although other values were tried in the course of iterative design.

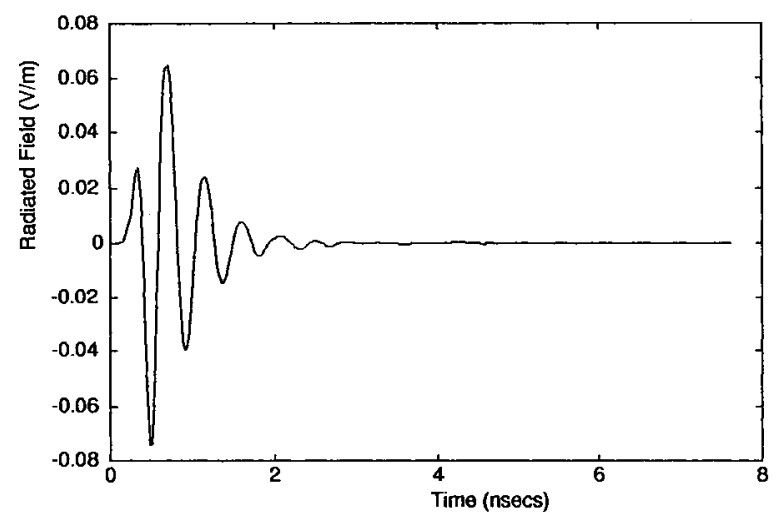

Fig. 6. Radiated dominant electric field component predicted by TDIE with 2 ns Gaussian input to element number 1 (the shortest) in the antenna shown in Fig. 5, at a distance of $2 \mathrm{~m}$ in the main-beam direction.

the feed network were substantially different from those that would be achieved with a traditional nose-fed LPDA (Table I).

The time delays appear somewhat counterintuitive, but they are less so if the "forward" and "reversed" driven dipoles are considered separately. Note that element number 7 has the minimum time delay: this is because number 1 was chosen as the reference element in the design process and this is in

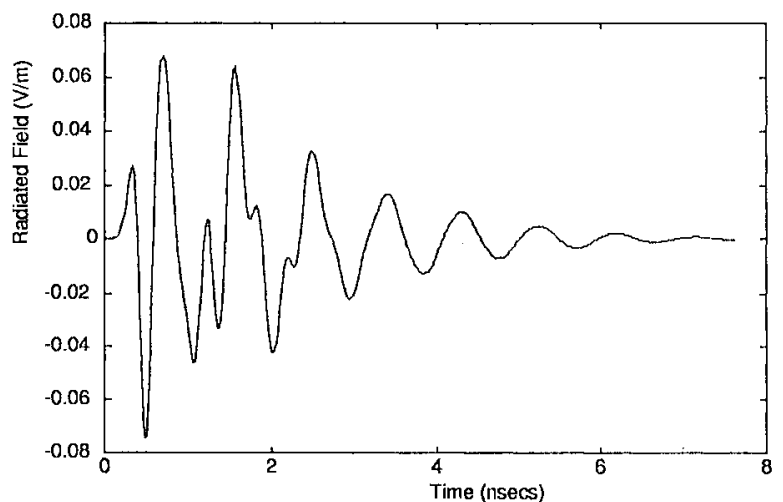

(a)

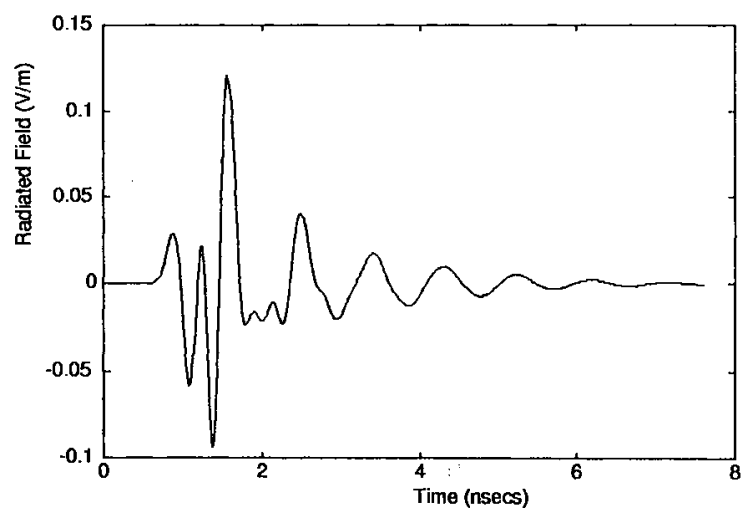

(b)

Fig. 7. Radiated dominant electric field component at $2 \mathrm{~m}$ from element number 1 in the main beam direction, predicted by TDIE. (a) With identical synchronized $2 \mathrm{~ns}$ Gaussian inputs to elements numbers 1 and 7 in the antenna shown in Fig. 5. (b) With the feed to element number 1 delayed to achieve coincidence of the two positive maxima in (a).

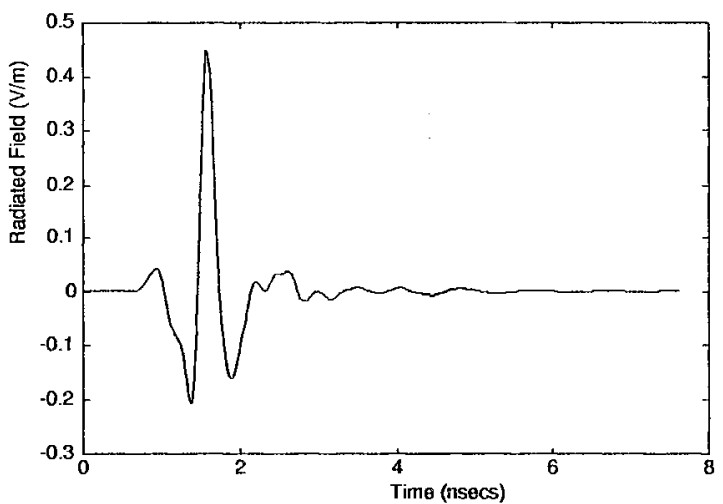

Fig. 8. Radiated dominant electric field component at $2 \mathrm{~m}$ from element number 1 in the main beam direction, predicted by TDIE with 2 ns Gaussian input to all elements in the antenna shown in Fig. 5, with time delays as given in Table I.

the "reversed" set. Number 7 is the member of this set that requires the least delay, while the whole of the "forward" set was found to require additional delay.

\section{REALIZATION OF THE NONDISPERSIVE LOG-PERIODIC ANTENNA}

Having devised this design, an attempt was then made to realize it in hardware. Two antennas were constructed-one 
TABLE I

Optimized Dimensions and Time Delays in the Feeds for the Dipoles of the Independently Fed Antenna Design (Fig. 5)

\begin{tabular}{c|c|c|c|c}
\hline Element No. & Dipole length, $\mathrm{mm}$ & Spacing from next, $\mathrm{mm}$ & Feed direction & Time delay, $\mathrm{ns}$ \\
\hline 1 & 123 & 8.4 & Reversed & 0.6532 \\
\hline 2 & 140 & 9.5 & Forward & 0.7821 \\
\hline 3 & 159 & 10.8 & Reversed & 0.4299 \\
\hline 4 & 180 & 12.3 & Forward & 0.6881 \\
\hline 5 & 205 & 14.0 & Reversed & 0.2441 \\
\hline 6 & 233 & 15.8 & Forward & 0.5735 \\
\hline 7 & 264 & 18.0 & Reversed & 0 \\
\hline 8 & 300 & - & Forward & 0.4034 \\
\hline
\end{tabular}

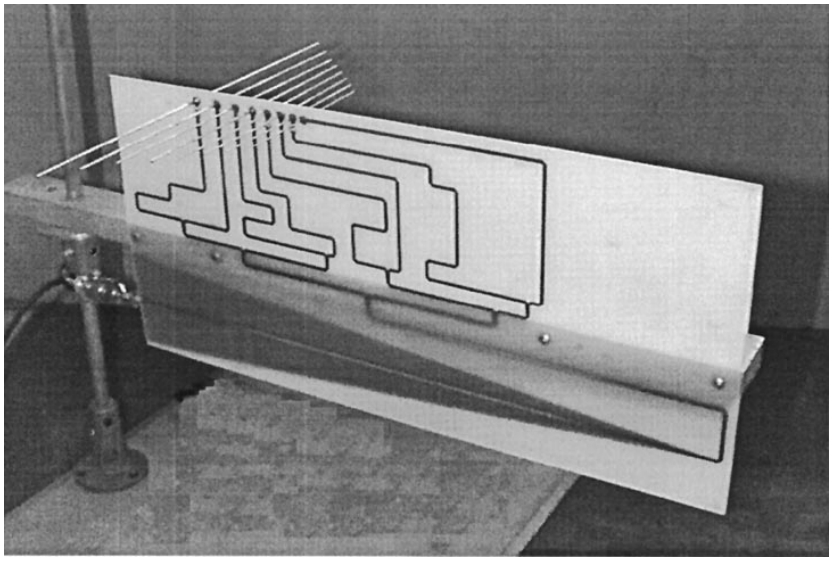

Fig. 9. The final antenna design, as realized with its normal-plane feed network.

according to the design derived above. The other had a more traditional value of the taper angle $\left(23^{\circ}\right)$ and with time delays in the individual feedlines chosen on an intuitive basis. In this case, the lengths of the separate element feed lines were arranged to be progressively reduced with increasing distance of the element from the nose such that the total time delay from the corporate feed point to each element and from that element to the nose was equalized.

Since the fields radiated by the antenna are everywhere normal to the plane that bisects the structure and is normal to the dipole elements, it was reasoned that a printed feed structure could be created in this plane that would not distort the fields and would not itself be disturbed by interaction with them. A sheet of printed circuit board material was thus used with the necessary transmission lines etched on both sides of the board to form balanced twin striplines. At the antenna elements requiring reverse-phase feed, two via holes were drilled and cross connections inserted at a break in the transmission lines. To enable the system to be fed from a coaxial transmission line, a broad-band balun was created by tapering one of the striplines so that it became very wide and, hence, gave the appearance of a ground plane. By using a long gentle taper it was found that a good broad-band performance could be achieved (Fig. 9). The effective permittivity of the stripline dielectric was taken into account in designing the length of the feedlines to achieve a given time delay. The lines were widened to give a halved characteristic impedance of $25 \Omega$ in a short section before each power division.

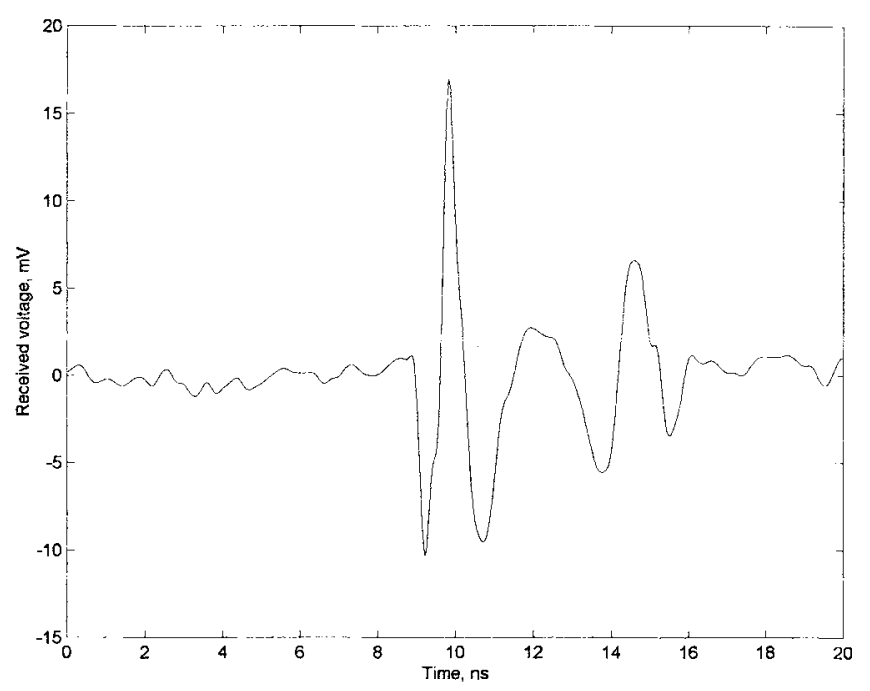

Fig. 10. Measured radiated electric field (dominant component) at $2 \mathrm{~m}$ from element number 1 in the main beam direction, with 2 ns Gaussian input to the antenna in Fig. 9.

In order to test these antennas, an avalanche-breakdown pulse generator was created as a source: this produced pulses with a rise-time of $400 \mathrm{ps}$. To probe the radiated fields, two bowtie dipole antennas were created with resistive end loading such that they were nonresonant [5]. Tests of these showed that they had a good deadbeat response with rise times much less than a nanosecond.

When the antenna designed by the procedure described above was tested it was found to give an excellent band-limited radiated pulse, the only corruption being a small time-delayed pulse about 5 ns later (Fig. 10). From its time delay, the latter is evidently a result of reflections at mismatches in the terminations of the feedline. The directive behavior of this antenna was investigated in a fully time-domain test and it was found that the antenna indeed showed directivity with much weaker and dispersed waveforms in directions away from the main beam (Fig. 11). When the second (intuitively-designed) log-periodic antenna was tested with the pulse generator and bowtie field probe dipole its pulsed performance was found not to be very satisfactory, with substantial dispersion of the frequency components (Fig. 12). This suggests either that some better paradigm for the intuitive design of such antennas is required or that intuitive design is infeasible. 

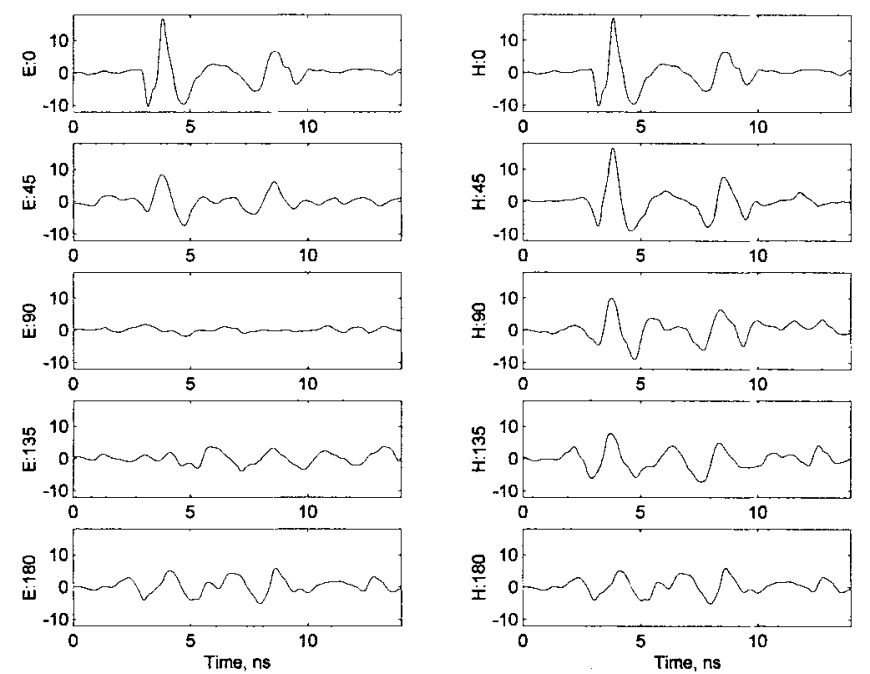

Fig. 11. Measured radiated electric field (dominant component) at $2 \mathrm{~m}$ from element number 1, demonstrating directive behavior in major selected directions. Vertical scale: V/m. Labels " $E: \theta$ " and " $H: \theta$ " refer to angle $\theta$ away from main beam direction in $E$-plane (containing the dipoles) and $H$-plane (normal to the dipoles), respectively. Only half of each plane shown as the other half is symmetrical.

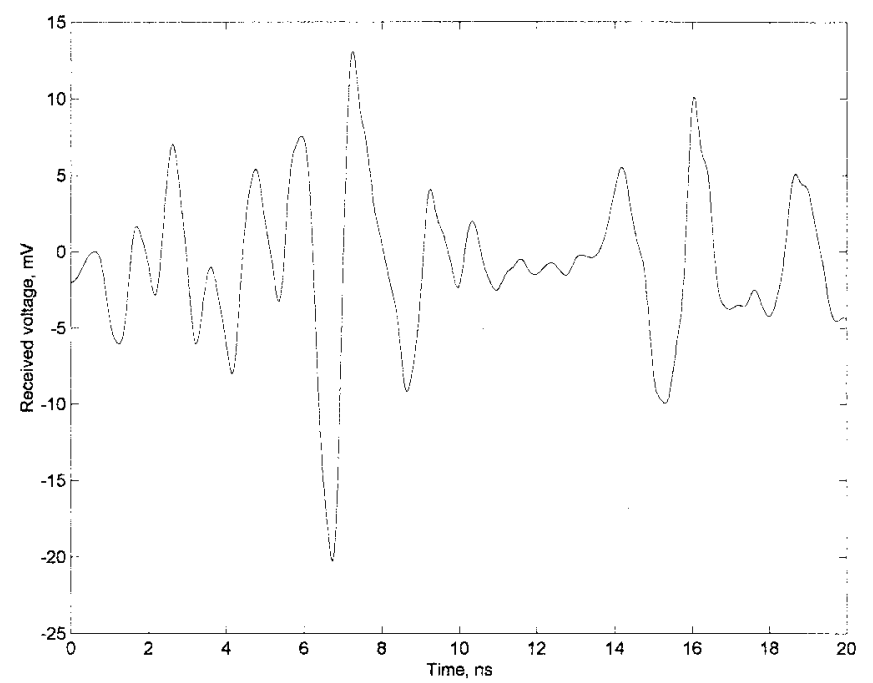

Fig. 12. Radiated electric field (dominant component) at $2 \mathrm{~m}$ from element number 1 , predicted by TDIE with 2 ns Gaussian input to all elements in a more conventional antenna structure with time delays chosen to compensate for the delays in lower-frequency elements.

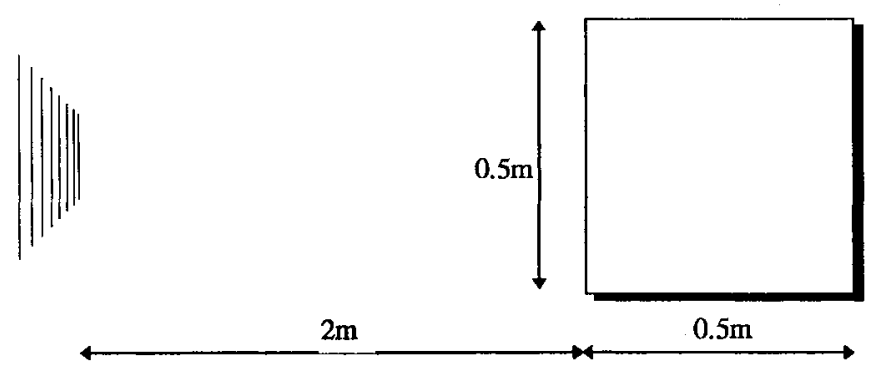

Fig. 13. Location of cubic test zone used to investigate quality of localized plane wave radiated from antenna.

It is considered that this novel nondispersive log-periodic antenna has many other applications, notably in wide-band ground-probing radar and in very wide-band communications,

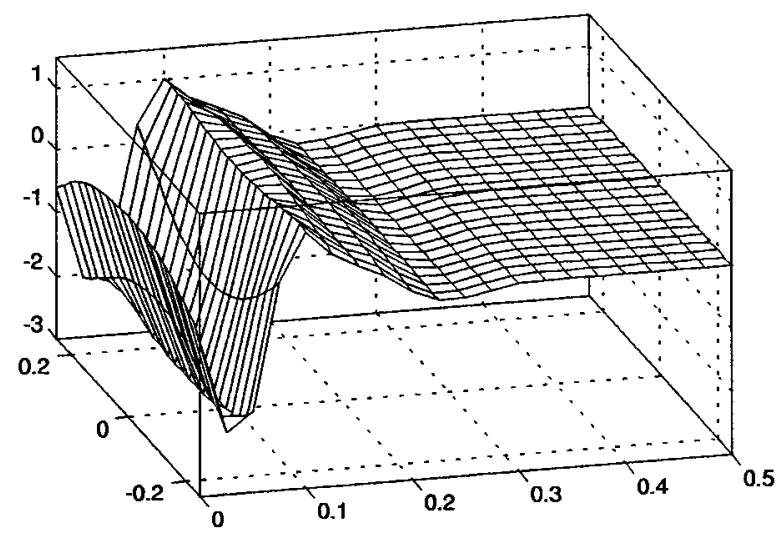

(a)

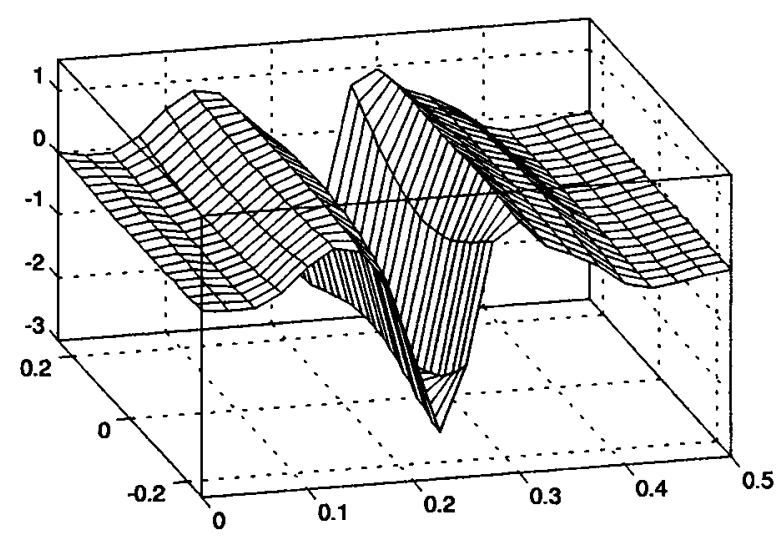

(b)

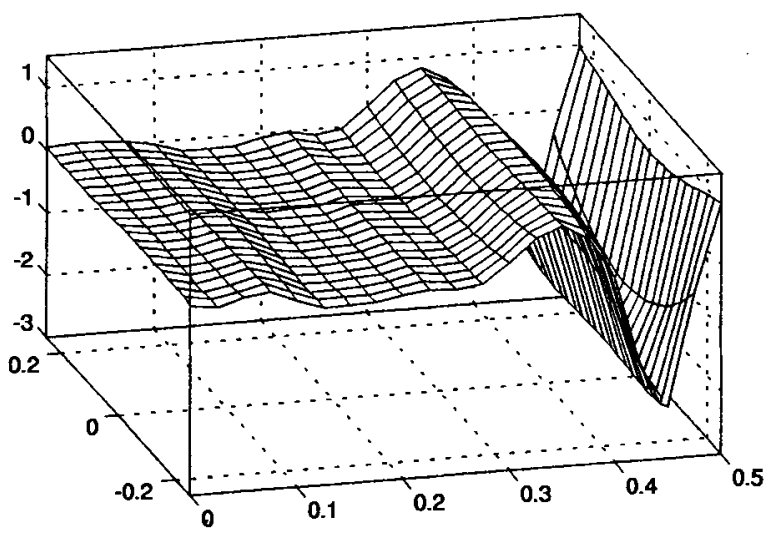

(c)

Fig. 14. Dominant component of a pulsed plane wave travelling across the test zone shown in Fig. 13, displayed at $0.7 \mathrm{~ns}$ intervals. Vertical scales: V/m; horizontal scales: m. (a) $t_{0}$. (b) $t_{0}+0.7 \mathrm{~ns}$. (c) $t_{0}+1.4 \mathrm{~ns}$.

where the dispersive nature of standard antennas would be unacceptable [6].

The arraying of the novel antenna design has not yet been tested, but computer simulation of the field distribution in a realistically sized test zone illuminated by the single nondispersive log-periodic antenna was undertaken (Fig. 13). The results (Fig. 14) show the dominant component of the electric field; the cross-polar component is inherently zero on the principal planes and will also be negligible for EMC purposes in off-axis directions within the zone examined. A 
plane wave of good quality is observed to be traveling through the zone: this demonstrates the potential for extension of the principle, e.g., by arraying to widen the zone.

\section{CONCLUSION}

Time-domain integral-equation (TDIE) studies of a trapezoidal log-periodic antenna of relatively standard form showed substantial dispersion in the radiated waveform when it was fed with a wide-band pulse. A radiated waveform much closer to a band-limited version of the pulse was achieved when the antenna was fed with a time-reversed replica of the dispersed waveform found in the first case.

A heuristic iterative design procedure was developed for a simple log-periodic dipole array (LPDA), based on TDIE modeling, which enabled the development of a design which was predicted to radiate a band-limited version of the input pulse without significant dispersion. This design differed from a conventional LPDA in that each element was fed by a separate source, each with an independently adjustable time delay. This contrasts with the standard transmission-line feeding method for LPDA's, which imposes inappropriate time delays that are proportional to the wavelength of the frequency component. The design was also unconventional in having relatively closely spaced elements leading to a very large value of the taper angle between the loci joining the tips of the dipole elements.

The novel design was realized in hardware, using a printed feed structure in the normal (bisecting) plane to create the desired independently adjustable time delays. A resistivelyloaded broad-band dipole antenna was used as a field probe and this showed that the novel LPDA could radiate a bandlimited pulse that corresponded reasonably well with the TDIE prediction. Tests of the radiated signals in directions away from the main beam showed that the antenna had significant directive behavior, displaying reduced amplitude and increased dispersion in directions away from the desired main beam.

A simulation of the use of such an antenna in an EMC test facility showed it to be capable of radiating a pulsed plane wave of good quality over a near-field test zone of useful size.

\section{REFERENCES}

[1] P. S. Excell, N. N. Jackson, and K. T. Wong, "A compact electromagnetic test range using an array of log-periodic antennas," Proc. Inst. Elect. Eng., vol. 140, pt. H, pp. 101-106, Apr. 1993.

[2] G. J. Burke, "Evaluation of modified log-periodic antennas for transmission of wide-band pulses," in Proc. 7th Annu. Rev. Appl. Computat. Electromagn., Monterey, CA, Mar. 1991, pp. 567-576.

[3] A. Tinniswood, A. M. Tyrrell, and S. R. Cloude, "Parallel implementation of time domain integral equation techniques in electromagnetics," in Proc. Eur. Electromagn. Compat. Conf., Zurich, Switzerland, 1993, pp. $503-508$.
[4] W. L. Stutzman and G. A. Thiele, Antenna Theory and Design. New York: Wiley, 1981, sec. 6.5

[5] R. W. Clarke and J. Rodriguez-Tellez, "A comparison of sheet metal and thick-film cavity backed bow-tie antennas," in Proc. 7th Int. Conf. Ground Penetrating Radar, Lawrence, KS, 1998, vol. 1, pp. 59-62.

[6] "Broadband Antenna," U.K. Patent Applicat. 97 26 816.3, 1997.

Peter S. Excell (M'80-SM'84) received the B.Sc. degree from the University of Reading, Reading, U.K., in 1970, and the Ph.D. degree from Bradford University, Bradford, U.K., in 1980, for research in electromagnetic hazards.

$\mathrm{He}$ is Professor of Applied Electromagnetics and Deputy Director of the Telecommunications Research Centre at the University of Bradford, where he has worked since 1971. He has been involved in research of electromagnetic compatibility since 1974, when he started work on an investigation of ignition hazards due to electromagnetic radiation. Later he initiated further research in compact range antennas for electromagnetic compatibility, probabilistic electromagnetic compatibility and superconducting filters and antennas. In 1991 he commenced a large-scale study of computational and measurement procedures for safety compliance testing of mobile phones. He is now a leading partner in a pan-European project to standardise such procedures across Europe. This has involved development of some of the most advanced electromagnetic models of the human body and of mobile phones using parallel computers to handle the high levels of detail and hybrid computational algorithms to enable conformal modeling of mobile phone details.

Dr. Excell is a Fellow of the IEE, a Chartered Engineer registered with the U.K. Engineering Council, and a member of the International Union of Radio Science (URSI), the Applied Computational Electromagnetics Society, the Bioelectromagnetics Society, and the European Bioelectromagnetics Association. He is also a member of the U.K. National Committee of URSI, representing Commission $\mathrm{K}$ (electromagnetics in medicine and biology) and a U.K. National Representative on the management committee of the European collaboration COST 261 (electromagnetic compatibility in large systems).

Adam D. Tinniswood received the B.Sc. and D.Phil. degrees from the Department of Electronics, University of York, U.K., in 1991 and 1996, respectively.

His doctoral thesis involved the implementation of time-domain integral equation electromagnetics algorithms on parallel computer architectures. He is currently a Staff Development Programmer with IBM Corporation, Server Development, Poughkeepsie, NY. From 1994 to 1996, he was a Research Assistant at the University of Bradford, U.K, working on the use of parallel computing for numerical simulations of the interaction of mobile telephones with the human head and on the design of impulse radiating antennas, using time-domain integral equations. In 1996 he moved to a Research Assistant Professorship in the Department of Electrical Engineering, University of Utah, Salt Lake City, where he expanded his activities in parallel computing for bioelectromagnetics. Since early 1999 he has been with IBM Corporation, working on development of high-availability server systems.

Roger W. Clarke (M'97) received the B.Sc. degree in mathematics and technology from the Open University, Milton Keynes, U.K., in 1993. He is currently working toward the Ph.D. degree in electronic materials for small antennas at Bradford University, Bradford, U.K.

$\mathrm{He}$ is a Senior Technical Officer in the Telecommunications Research Centre, University of Bradford, U.K., where he has worked since 1987. His research interests include analysis of small antennas and semiconductor devices for RF and power applications.

Mr. Clarke is an associate member of the IEE. 